\title{
Histoire de la Kétamine : une molécule ancienne qui a toujours la cote
} History of Ketamine: an ancient molecule that is still popular today

\author{
Brendan Le Daré ${ }^{1,2}$, Romain Pelletier ${ }^{2}$, Isabelle Morel $^{1,2}$, Thomas Gicquel ${ }^{1,2}$ \\ ${ }^{1}$ Univ Rennes, INSERM, INRAE, Institut NuMeCan (Nutrition, Métabolismes et Cancer), F- \\ 35000 Rennes, France \\ ${ }^{2}$ Laboratoire de Toxicologie biologique et médico-légale, Centre Hospitalier Universitaire \\ Pontchaillou, F-35000 Rennes, France
}

Auteur correspondant : Dr. Brendan Le Daré (brendan.le.dare@chu-rennes.fr)

ORCID : 0000-0002-5907-2450

Publons: AAL-7050-2020

Adresse : Laboratoire de Toxicologie Biologique et Médico-légale, Centre Hospitalier Universitaire Pontchaillou, 2 Rue Henri Le Guilloux, 35000 Rennes, France 


\section{Résumé}

L'histoire de la kétamine commence en 1962, lorsque Calvin Stevens du laboratoire pharmaceutique Parke-Davis la synthétise à partir de la phencyclidine, une molécule aux propriétés psychodysleptiques hallucinogènes et dissociatives. Suite à la première administration de kétamine chez l'homme en 1964 à la prison Jackson (Michigan, USA), ses effets dissociatifs associés à l'obtention de l'anesthésie courte sont rapportés, et un brevet portant sur son utilisation humaine est déposé en 1966. Dans les années 1990, la découverte de l'hyperalgie induite par les opioïdes suscite un intérêt pour la kétamine dans l'indication analgésique. Ces dernières années, l'utilisation humaine de la kétamine, et en particulier son énantiomère eskétamine, s'est orienté dans la prise en charge de la dépression. Les premiers cas d'abus de kétamine sont signalés en 1992 en France, menant à une surveillance particulière par les autorités sanitaires, et son inscription en 1997 sur la liste des stupéfiants. Aujourd'hui, la kétamine est devenue une substance attractive en usage récréatif, émergeant peu à peu des milieux techno alternatifs pour se répandre sur les scènes festives plus commerciales. Ces éléments représentent une préoccupation de santé publique, associé au 
risque de développement de nouveaux analogues de synthèse chimique dont les effets néfastes sont encore peu connus.

\section{Abstract}

The history of ketamine begins in 1962, when Calvin Stevens of the pharmaceutical laboratory Parke-Davis synthesizes it from phencyclidine, a molecule with psychodysleptic, hallucinogenic and dissociative properties. Following the first administration of ketamine to humans in 1964 in Jackson prison (Michigan, USA), its dissociative effects associated with short anaesthesia were reported, and a patent for its human use was filed in 1966. In the 1990s, the discovery of opioid-induced hyperalgesia sparked interest in ketamine as an analgesic. In recent years, the human use of ketamine, and in particular its esketamine enantiomer, has shifted towards the treatment of depression. The first cases of ketamine abuse were reported in 1992 in France, leading to special surveillance by the health authorities, and its inclusion in the list of narcotic drugs in 1997. Today, ketamine has become an attractive substance for recreational use, gradually emerging from alternative techno circles to spread to more commercial party scenes. These elements represent a public health concern, associated with the risk of developing new chemically synthesized analogues, the harmful effects of which are still little known.

Mots clés : kétamine, anesthésie, analgésie, dépression, usage récréatif 
Keywords: ketamine, anesthesia, analgesia, depression, recreational use

\section{Points essentiels :}

- L'histoire de la kétamine commence en 1962, lorsqu'elle est synthétisée à partir de la phencyclidine

- La kétamine est une molécule psychotrope utilisée dans des indications d'anesthésiologie, d'analgésie et de traitement des épisodes dépressifs caractérisés.

- La relative sécurité d'emploi et l'effet dissociatif de la kétamine en ont fait une substance attractive en usage récréatif, se répandant sur les scènes festives commerciales.

- L'apparition d'analogues de synthèse de la kétamine ces dernières années représente une préoccupation de santé publique

\section{Introduction}


La kétamine appartient à la classe chimique des «phenylcyclohexylamines ». Elle est dérivée de la phencyclidine (PCP), molécule possédant des propriétés psychodysleptiques hallucinogènes et dissociatives qui entrainent un potentiel d'abus important [1]. La kétamine se présente sous forme de cristaux blancs dont la structure chimique consiste en un mélange racémique de $(S)$-kétamine et $(R)$-kétamine (Figure 1). Cette substance est un puissant anesthésique aux propriétés dissociatives, permettant l'obtention d'une anesthésie sans complète inconscience, caractérisée par une catatonie, une cataplexie, et une amnésie. Son absence d'effet dépresseurs respiratoire et sa demi-vie courte en font une substance de choix en thérapeutique. Également, cette molécule exerce des effets analgésiques, antiinflammatoires et antidépresseurs [1]. Cependant, du fait de son détournement d'usage à but récréatif, cette molécule est classée en France comme stupéfiant depuis 1997 [2]. D’abord développée dans les années 1960 à visée thérapeutique, son détournement est de plus en plus important aujourd'hui, sortant des milieux techno alternatifs, pour se répandre sur les scènes festives plus commerciales (clubs, bars) [3].

\section{Propriétés et mode d'action de la kétamine}

A la suite d'une administration orale, l'absorption de la kétamine est rapide, mais sa biodisponibilité est faible, de l'ordre de 8 à $24 \%$. En termes de distribution, la kétamine est faiblement liée aux protéines plasmatiques, et franchit aisément la barrière hématoencéphalique. L'obtention des effets antalgiques et anesthésiques est donc rapide et durent plusieurs heures [4]. Le métabolisme important de la kétamine, passant par les cytochromes P450 (CYP450) 3A et 2B6, explique son effet de premier passage hépatique et son éventuelle modulation par des interactions médicamenteuses [5,6]. Du fait de ses propriétés hydrosolubles, peu de métabolites sont produits, conduisant principalement à la norkétamine 
(métabolite actif), et à des dérivés hydroxylés [1,7]. Ces métabolites, qui subissent une glucuroconjugaison, sont ensuite éliminés par voie biliaire et urinaire [8]. En termes d'élimination, sa demi-vie plasmatique est de 2 à 4 heures, permettant un réveil également rapide en cas d'indication en anesthésiologie [5].

D'un point de vue pharmacologique, la kétamine cible plusieurs récepteurs du système nerveux central. Contrairement à d'autres anesthésiques, elle n'affecte pas les récepteurs à l'acide gamma-aminobutyrique (GABA), mais est antagoniste des récepteurs N-Méthyl-DAspartate (NMDA) au glutamate dans le cerveau et la moelle épinière $[9,10]$. En particulier, l'énantiomère $\mathrm{S}$ de la kétamine présente une meilleure affinité ainsi qu'une activité quatre fois plus puissante que l'énantiomère R envers les récepteurs NMDA. La kétamine potentialiserait ainsi l'inhibition descendante de la moelle épinière dans les pathologies douloureuses chroniques $(6,11)$. De plus, la kétamine possède une activité agoniste des récepteurs opioïdes $\mu$ et $\kappa$, là aussi avec une affinité plus puissante de l'énantiomère $S$ en comparaison du mélange racémique [11]. Complémentairement, un effet inhibiteur de la recapture des catécholamines neuronale et extra-neuronale a été décrit, impliquant particulièrement la dopamine. Enfin, la kétamine possède un effet inhibiteur des récepteurs muscariniques M1 et diminue la durée d'ouverture du canal associé aux récepteurs nicotiniques [12]. De ce fait, la diversité des récepteurs interagissant avec la kétamine est à l'origine de la complexité des effets cliniques retrouvés.

La kétamine augmente la pression artérielle et le rythme cardiaque, tout en préservant l'activité respiratoire, rendant ainsi un surdosage mortel peu probable. A dose modérée, elle provoque des effets euphorisants et une ébriété «cotonneuse »; des hallucinations mais aussi des effets dissociatifs (sensation de rupture de l'unité psychique et corporelle) surviennent à 
plus forte dose. La prise de kétamine provoque un engourdissement avec perte du sens de l'espace, un sentiment de dissociation entre le corps et l'esprit. Elle peut être à l'origine de pertes de connaissance voire de coma, en particulier en association avec l'alcool [13]. Cette symptomatologie, dénommée «K-hole » du fait d'un effet «trou noir » est caractéristique de la kétamine et entraine des troubles cognitifs et amnésiques, avec troubles de l'humeur et du comportement, délires hallucinatoires, cauchemars, perte d'identité et du contact à la réalité.

En termes d'effets indésirables, l'usage chronique de kétamine a été associé à des désordres urinaires sévères, tels que des cystites, hyperréactivité vésicales et un épaississement de la paroi vésicale [14]. Des lésions rénales secondaires, parfois irréversibles ont été rapportées [15]. Une toxicité gastro-intestinale est également décrite dans la littérature sous le nom de «K-pain », allant d'une simple douleur abdominale à d'intenses coliques abdominales [16,17]. Des pathologies hépatiques, telles que des anomalies des bilans hépatiques, des kystes cholédoques et des dilatations du canal cholédoque ont été décrits [18,19]. En 2017, l'ANSM rapportait ainsi le signalement de dix cas d'atteintes hépatiques graves survenues depuis 2014, et susceptibles d'être liées à l'utilisation répétée et/ou prolongée de kétamine (entre 1 mois et 5 mois de traitement continu) à doses élevées (dépassant 100 mg/jour). Parmi celles-ci, quatre patients avaient dû subir une transplantation hépatique [20]. Enfin, la tolérance à la kétamine est connue depuis de nombreuses années chez l'homme [21]. Cependant, très peu de cas de syndrome de sevrage ont été décrits [19]. Bien que rares, des cas de dépendance à la kétamine ont également été rapportés [22-25].

\section{Historique de la kétamine}


Pour comprendre l'histoire de la kétamine, il est nécessaire de s'intéresser à celle de son analogue chimique, la phencyclidine (PCP). Cette dernière, synthétisée en 1956 par Harnold V. Maddox du laboratoire pharmaceutique Parke-Davis (Michigan, USA), constitue la première molécule issue des recherches visant à trouver un nouvel anesthésique doté de propriétés analgésiques parmi les cyclohexylamines [26]. Deux ans plus tard (1958) les premiers essais de la PCP, sous le nom de Sernyl®, étaient réalisés chez l'homme [27]. Cependant, les réactions parfois incontrôlables qualifiées de «psychotiques » en période postopératoire pour $15 \%$ des patients menèrent à l'abandon de cette molécule à parti de 1965 , au profit de la kétamine [28,29]. Le Sernyl®, PCP, doté de propriétés psychédéliques mais délaissé par le corps médical, devient alors une drogue de rue, appelée «poussière d'ange » $[30]$.

L'histoire de la kétamine commence ainsi en 1962, lorsque Calvin Stevens, du laboratoire pharmaceutique Parke-Davis également, la synthétise à partir de la phencyclidine [4,31]. Suite à sa première administration chez l'homme en 1964 à la prison Jackson (Michigan, USA), ses effets dissociatifs associés à l'obtention de l'anesthésie courte sont rapportés, et un brevet portant sur son utilisation humaine est déposé en 1966 [32]. Sa faible fréquence d'effets indésirables, associée à l'absence d'émergence de comportement psychotique comparé à la phencyclidine participeront grandement à son implantation dans les protocoles de soins. En 1970, la Food and Drug Administration (FDA) autorisait la mise sur le marché du Ketalar®, première préparation de kétamine à usage humain [33]. Pendant la guerre du Vietnam (19551975), la kétamine était l'analgésique le plus utilisé sur les champs de bataille [34]. De manière plus marginale, cette substance fût également utilisée par Karl Jansen, psychiatre néo-zélandais, dans le traitement des addictions et autres maladies mentales [29]. En 1989, l'autorisation de mise de mise sur le marché (AMM) du propofol par la FDA conduit à 
diminuer peu à peu l'utilisation de la kétamine en anesthésie [35]. De manière intéressante, la découverte de l'hyperalgie induite par les opioïdes en 1990 conduit à des changements de pratique, et provoque un regain d'intérêt pour la kétamine dans l'indication analgésique. Ces dernières années, l'utilisation humaine de la kétamine semble également s'orienter dans la prise en charge de la dépression [36].

\section{Usage actuel de la kétamine}

\subsection{Usages thérapeutiques de la kétamine}

En France, la kétamine possède une AMM dans diverses indications d'anesthésiologie et psychiatrie mais est également utilisée dans des indications hors AMM principalement pour ses propriétés antalgiques (Tableau 1).

L'indication la plus utilisée de la kétamine reste sans conteste l'anesthésie du fait de son effet rapide et de sa bonne tolérance. En particulier, ses effets activateurs du système sympathique en font une substance de choix chez les patients présentant une instabilité hémodynamique tels que les patients polytraumatisés, ou en choc septique. Également, l'utilisation possible de la kétamine en intramusculaire présente un avantage considérable chez les patients pour lesquels une administration intraveineuse se présente difficile. La population pédiatrique représente dans ce contexte une indication privilégiée, faisant de la kétamine une des substances les plus utilisées en sédation ou anesthésie dans les services d'urgence pédiatrique [26]. Enfin, il est intéressant de citer le cas des patients traumatisés crâniens, pour lesquels la kétamine a montré des effets neuroprotecteurs et un bon profil de tolérance en cas de risque d'hypertension intracrânienne [37,38]. En termes de posologie, une induction de l'anesthésie 
est généralement obtenue par voie intraveineuse en bolus ou perfusions rapides de 1 à 5 $\mathrm{mg} / \mathrm{kg}$ ou par voie intramusculaire de 6,5 à $13 \mathrm{mg} / \mathrm{kg}$. La posologie d'entretien consiste alors à la réinjection d'une dose comprise entre la moitié et la totalité de la dose nécessaire pour l'induction par voie intraveineuse ou intramusculaire selon les besoins [39]. L'énantiomère S, dénommé eskétamine, possède une activité anesthésique et antalgique deux à quatre fois plus importante que le mélange racémique, et présente moins d'effets indésirables de type psychodysleptiques [5]. Cependant, l'eskétamine en injectable n'est pas commercialisée en France, contrairement à certains pays comme les Pays-Bas, l'Allemagne et la Norvège où elle est utilisée comme anesthésiant (Ketanest $\left.{ }^{\circledR}\right)$.

Dans les douleurs aigues, l'association de kétamine à des opioïdes a montré une diminution de leurs effets dépresseurs respiratoires, une diminution de l'hyperalgie induite par les opioïdes, et une diminution des consommations d'antalgiques en post-opératoire [40-42]. Également, des doses sub-dissociatives de $0,3 \mathrm{mg} / \mathrm{kg}$ ont montré une efficacité et une tolérance comparable à l'utilisation intraveineuse de morphine à court terme [43]. Ces propriétés ont notamment argumenté l'utilisation de kétamine dans des indications hors AMM telles que les douleurs aigues postopératoires ou les douleurs aigues aux urgences et en réanimation, notamment pour la réalisation de soins douloureux (Tableau 1). De manière intéressante, la kétamine présente également des propriétés anti-inflammatoires, pouvant en partie contribuer aux effets antalgiques observés [44,45].

Dans les douleurs chroniques, l'utilisation de la kétamine n'est pas récente, ayant ainsi amené l'AFSSAPS à établir des recommandations de bonnes pratiques de 2010 «en situation palliative avancée », malgré l'absence d'AMM en analgésie [46]. Dans le cadre des douleurs chroniques rebelles, de nombreuses études se sont penchées sur l'intérêt de la kétamine en 
particulier dans le cas des douleurs neuropathiques. Des formulations nasales et sublinguales ont été développées en Australie, permettant de proposer des alternatives à l'injection intraveineuse dans le traitement de ces affections [5,47]. Niesters et al. (2013) rapportent l'utilisation de la kétamine dans les douleurs migraineuses, les douleurs cancéreuses, les neuropathies induites par les chimiothérapies, la fibromyalgie, les douleurs ischémiques des membres, les douleurs des membres fantômes, les douleurs post-zostériennes ou encore les douleurs par lésion traumatique nerveuse [48]. Cependant, la littérature reste très hétérogène quant aux posologies, durées et fréquences d'administration utilisées. Selon la Société Française d'Anesthésie et de Réanimation, la concentration plasmatique efficace de la kétamine pour l'analgésie se situe entre 20 et $100 \mathrm{ng} / \mathrm{mL}$, nécessitant l'administration intraveineuse de bolus de $0,15 \mathrm{mg} / \mathrm{kg}$ à $0,5 \mathrm{mg} / \mathrm{kg}$ répétés et/ou la perfusion continue de 0,125 à $0,25 \mathrm{mg} / \mathrm{kg} / \mathrm{h}$ [49]. A défaut de recommandations françaises, seules des recommandations américaines publiées en juillet 2018 font référence actuellement [50].

Dans le traitement des épisodes dépressifs caractérisés, la notion de stéréosélectivité de la kétamine est de plus en plus récurrente dans la littérature [51]. En particulier, de récentes études ont montré l'intérêt de l'eskétamine chez les patients résistants aux traitements conventionnels, avec un délai d'action de quelques heures [52]. Cet énantiomère a ainsi été commercialisée en France sous forme de pulvérisation nasale sous le nom de Spravato® depuis décembre 2019 dans le traitement des épisodes dépressifs caractérisés résistants n'ayant pas répondu à au moins deux antidépresseurs différents au cours de l'épisode dépressif actuel modéré à sévère (Tableau 1). Cette spécialité a cependant été classée parmi les molécules à éviter en 2021 par une revue de médecine française du fait d'une efficacité incertaine, couplée à la survenue d'effets indésirables tels que des syndromes de dissociation chez $60 \%$ à $79 \%$ des patients (hallucinations, confusions, distorsions du temps et de l'espace, 
altérations sensorielles, sensations d'être en dehors de son propre corps), des effets sédatifs allant jusqu'à la perte de conscience avec dépression respiratoire ou des poussées hypertensives [53].

\subsection{Usages récréatifs de la kétamine}

En France, les premiers cas d'abus de kétamine ont été signalés en 1992, menant à une surveillance particulière par les autorités sanitaires [54]. En 1997, la kétamine et ses sels sont inscrits sur la liste des stupéfiants, bien que les préparations injectables restent inscrites sur la liste I des substances vénéneuses. Du fait de l'augmentation des cas d'abus à partir de 2002 rapportés par les Centre d'Evaluation et d'Information sur la Pharmacodépendance, ces préparations injectables de kétamine rejoindront la liste des stupéfiants le 24 avril 2017. Aujourd'hui, la prescription de kétamine est donc requise sur ordonnance sécurisée uniquement par des médecins spécialisés en anesthésie-réanimation ou en médecine d'urgence, imposant également la traçabilité des entrées et sorties sur un registre spécial.

Dans le cadre de son usage récréatif, la kétamine est connue sous différents noms, tels que : «Kéta », «Ké », « Kéké », « Ket », «Spécial K », « Kit Kat », « Kate », « Keller », «Vitamine $\mathrm{K} »$, «Super acide », « Super $\mathrm{C} »$ ou encore « drogue à poney » [13]. On la trouve sous forme liquide ou «cuisinée » sous forme de poudre. En France, la hausse de la disponibilité en kétamine ces dernières années est portée par le développement de microtrafics d'usagers-revendeurs s'approvisionnant auprès de réseaux des pays transfrontaliers (PaysBas, Belgique et Espagne), ainsi que par les achats sur le darknet. Elle se retrouve ainsi systématiquement disponible en free party et dans une moindre mesure dans les soirées urbaines en clubs et bars [3,55]. Ce «succès» de la kétamine s'explique en partie par 
l'absence de détection de cette substance par les tests rapides réalisés en bord de route par les forces de l'ordre. En termes de prix, le gramme de kétamine est généralement vendu entre 40 et 50 euros [56]. De manière intéressante, le mésusage de kétamine semble également avoir gagné en popularité de certains pays asiatiques ces dernières années. Pour l'exemple d'Hong Kong, la kétamine y semble être devenue une des substances les plus régulièrement consommées à but récréatif. Depuis 2017, certains laboratoires de Toxicologie d'Hong Kong rapportent également l'apparition d'analogues de la kétamine, tels que la deschloro-N-ethylkétamine (2-oxo-PCE) [57]. Cet analogue a été récemment mis en évidence en France [58]. Cependant, bien que ces cas ne concernent que la région de Hong Kong, D’autres dérivés de la kétamine, comme la N-ethyl-kétamine ou la 2-méthoxy-kétamine, semblent également arriver en Europe [59].

Au-delà des usages récréatifs, des cas de soumission chimique impliquant la kétamine ont été rapportés. Dans ce contexte, ce sont les effets amnésiants associés à l'altération des fonctions motrices qui sont détournés à des fins criminelles [60]. Du fait de son absence d'odeur, de saveur et de sa forte solubilité dans l'eau, sa détection par la victime est extrêmement difficile. De plus, son élimination rapide n'offre qu'une fenêtre de détection très courte pour les analyses toxicologiques. Des méthodes analytiques de pointes sont donc primordiales dans le but d'objectiver toute consommation [61]. La kétamine est fréquemment mise en évidence dans les cheveux de consommateurs chroniques de drogues. Ainsi, dans une série française de 141 sujets montrant la présence de nouvelles substances psychoactives dans les cheveux, la kétamine est retrouvée chez $67 \%$ des sujets [62].

\section{Quelles perspectives thérapeutiques pour la kétamine en France?}


Dans la littérature, l'identification des cibles pharmacologiques responsables des différents effets de la kétamine semble constituer un enjeu important dans le développement de nouvelles perspectives thérapeutiques présentant moins d'effets indésirables et moins de potentiels d'abus. En particulier, l'exploration des effets cliniques de la S-kétamine, la Rkétamine et de son métabolite actif norkétamine pourrait constituer les bases d'optimisation des prises en charge des patients douloureux, des états dépressifs caractérisés et de certaines pathologies inflammatoires.

\section{Conclusion}

Depuis sa découverte en 1962, la kétamine s’est imposée comme un précieux anesthésique, en particulier chez les patients à l'hémodynamique instable et dans la population pédiatrique. D'autres indications ont également émergé telles que l'analgésie et le traitement de la dépression. Cependant, sa relative sécurité d'emploi et son effet dissociatif en ont fait une substance attractive en usage récréatif, émergeant peu à peu des milieux techno alternatifs, pour se répandre sur les scènes festives plus commerciales. Ces éléments représentent une préoccupation de santé publique et risquent de stimuler le développement de nouveaux analogues de synthèse chimique dans l'objectif d'augmenter les effets psychodysleptiques, au détriment de la sécurité d'emploi.

\section{Déclaration d'intérêt}

Les auteurs ont déclaré n'avoir aucun conflit d'intérêt en lien avec cet article.

\section{REFERENCES}


[1] Zanos P, Moaddel R, Morris PJ, Riggs LM, Highland JN, Georgiou P, et al. Ketamine and Ketamine Metabolite Pharmacology: Insights into Therapeutic Mechanisms. Pharmacol Rev 2018;70:621-60. https://doi.org/10.1124/pr.117.015198.

[2] Arrêté du 8 août 1997 modifiant l'arrêté du 22 février 1990 fixant la liste des substances classées comme stupéfiants. n.d.

[3] Observatoire français des drogues et toxicomanies. Usagers, marchés et substances : évolutions récentes (2018 - 2019) n.d. https://www.ofdt.fr/BDD/publications/docs/eftxcgzc.pdf (accessed September 1, 2020).

[4] Domino EF. Taming the Ketamine Tiger: Anesthesiology 2010:1. https://doi.org/10.1097/ALN.0b013e3181ed09a2.

[5] Peltoniemi MA, Hagelberg NM, Olkkola KT, Saari TI. Ketamine: A Review of Clinical Pharmacokinetics and Pharmacodynamics in Anesthesia and Pain Therapy. Clin Pharmacokinet 2016;55:1059-77. https://doi.org/10.1007/s40262-016-0383-6.

[6] Peltoniemi MA, Saari TI, Hagelberg NM, Laine K, Kurkinen KJ, Neuvonen PJ, et al. Rifampicin has a Profound Effect on the Pharmacokinetics of Oral S-Ketamine and Less on Intravenous SKetamine. Basic Clin Pharmacol Toxicol 2012;111:325-32. https://doi.org/10.1111/j.17427843.2012.00908.x.

[7] Hashimoto K. Molecular mechanisms of the rapid-acting and long-lasting antidepressant actions of (R)-ketamine. Biochem Pharmacol 2020;177:113935. https://doi.org/10.1016/j.bcp.2020.113935.

[8] Mion G, Villevieille T. Ketamine Pharmacology: An Update (Pharmacodynamics and Molecular Aspects, Recent Findings). CNS Neurosci Ther 2013;19:370-80. https://doi.org/10.1111/cns.12099.

[9] Salmi E, Langsjö JW, Aalto S, Nagren K, Metsahonkala L, Kaisti KK, et al. Subanesthetic Ketamine Does Not Affect 11C-Flumazenil Binding in Humans: Anesth Analg 2005;101:722-5. https://doi.org/10.1213/01.ANE.0000156951.83242.8D.

[10] Zorumski CF, Izumi Y, Mennerick S. Ketamine: NMDA Receptors and Beyond. J Neurosci 2016;36:11158-64. https://doi.org/10.1523/JNEUROSCI.1547-16.2016.

[11] Hustveit O, Maurset A, Oye I. Interaction of the chiral forms of ketamine with opioid, phencyclidine, sigma and muscarinic receptors. Pharmacol Toxicol 1995;77:355-9. https://doi.org/10.1111/j.1600-0773.1995.tb01041.x.

[12] Kohrs R, Durieux ME. Ketamine: teaching an old drug new tricks. Anesth Analg 1998;87:118693. https://doi.org/10.1097/00000539-199811000-00039.

[13] Gandilhon M, Cadet-Taïrou A, Martinez M. L'usage de kétamine en France : tendances récentes (2012-2013) n.d.:8.

[14] Winstock AR, Mitcheson L, Gillatt DA, Cottrell AM. The prevalence and natural history of urinary symptoms among recreational ketamine users: urinary symptoms in ketamine users. BJU Int 2012;110:1762-6. https://doi.org/10.1111/j.1464-410X.2012.11028.x.

[15] Chu PS-K, Ma W-K, Wong SC-W, Chu RW-H, Cheng C-H, Wong S, et al. The destruction of the lower urinary tract by ketamine abuse: a new syndrome? BJU Int 2008;102:1616-22. https://doi.org/10.1111/j.1464-410X.2008.07920.x.

[16] Jansen KLR. A Review of the Nonmedical Use of Ketamine: Use, Users and Consequences. J Psychoactive Drugs 2000;32:419-33. https://doi.org/10.1080/02791072.2000.10400244.

[17] Morgan CJA, Curran HV, the Independent Scientific Committee on Drugs (ISCD). Ketamine use: a review: Ketamine use: a review. Addiction 2012;107:27-38. https://doi.org/10.1111/j.1360-0443.2011.03576.x.

[18] Wong SW, Lee KF, Wong J, Ng WWC, Cheung YS, Lai PBS. Dilated common bile ducts mimicking choledochal cysts in ketamine abusers. Hong Kong Med J Xianggang Yi Xue Za Zhi 2009;15:53-6.

[19] Sassano-Higgins S, Baron D, Juarez G, Esmaili N, Gold M. A REVIEW OF KETAMINE ABUSE AND DIVERSION: Review: Ketamine. Depress Anxiety 2016;33:718-27. https://doi.org/10.1002/da.22536.

[20] Agence Nationale de Sécurité du Médicaments et des produits de santé. Kétamine : risque d'atteintes hépatiques graves lors d'utilisations prolongées et/ou à doses élevées - Point d'Information 2017. 
[21] Byer DE, Gould AB. Development of Tolerance to Ketamine in an Infant Undergoing Repeated Anesthesia: Anesthesiology 1981;54:255-6. https://doi.org/10.1097/00000542-19810300000016.

[22] Cheng W-J, Chen C-H, Chen C-K, Huang M-C, Pietrzak RH, Krystal JH, et al. Similar psychotic and cognitive profile between ketamine dependence with persistent psychosis and schizophrenia. Schizophr Res 2018;199:313-8. https://doi.org/10.1016/j.schres.2018.02.049.

[23] Garg A, Sinha P, Kumar P, Prakash O. Use of naltrexone in ketamine dependence. Addict Behav 2014;39:1215-6. https://doi.org/10.1016/j.addbeh.2014.04.004.

[24] Pal HR, Berry N, Kumar R, Ray R. Ketamine dependence. Anaesth Intensive Care 2002;30(3):382-4. https://journals.sagepub.com/doi/10.1177/0310057X0203000323.

[25] Hurt PH, Ritchie EC. A case of ketamine dependence. Am J Psychiatry 1994;151:779a-79. https://doi.org/10.1176/ajp.151.5.779a.

[26] Li L, Vlisides PE. Ketamine: 50 Years of Modulating the Mind. Front Hum Neurosci 2016;10. https://doi.org/10.3389/fnhum.2016.00612.

[27] Rudgley R. The encyclopedia of psychoactive substances. Macmillan 2014.

[28] Johnstone M, Evans V, Baigel S. Sernyl@ (C1-395) in clinical anaesthesia. Br J Anaesth 1959;31:433-9. https://doi.org/10.1093/bja/31.10.433.

[29] Mion G. Histoire de la kétamine et du psychédélisme. Ann Méd-Psychol Rev Psychiatr 2017;175:661-4. https://doi.org/10.1016/j.amp.2017.07.002.

[30] Bertron JL, Seto M, Lindsley CW. DARK Classics in Chemical Neuroscience: Phencyclidine (PCP). ACS Chem Neurosci 2018;9:2459-74. https://doi.org/10.1021/acschemneuro.8b00266.

[31] Copeland J, Dillon P. The health and psycho-social consequences of ketamine use. Int J Drug Policy 2005;16:122-31. https://doi.org/10.1016/j.drugpo.2004.12.003.

[32] Dundee JW. Twenty-five years of ketamine A report of an international meeting. Anaesthesia 1990;45:159-60. https://doi.org/10.1111/j.1365-2044.1990.tb14287.x.

[33] Ketalar. "U.S. food and drug administration, center for drug evaluation and research," in Label and Approval History. 1970.

[34] Orhurhu VJ, Vashisht R, Cohen SP. Ketamine Toxicity. StatPearls, Treasure Island (FL): StatPearls Publishing; 2020.

[35] Chidambaran V, Costandi A, D'Mello A. Propofol: a review of its role in pediatric anesthesia and sedation. CNS Drugs 2015;29:543-63. https://doi.org/10.1007/s40263-015-0259-6.

[36] Nowacka A, Borczyk M. Ketamine applications beyond anesthesia - A literature review. Eur J Pharmacol 2019;860:172547. https://doi.org/10.1016/j.ejphar.2019.172547.

[37] Bar-Joseph G, Guilburd Y, Tamir A, Guilburd JN. Effectiveness of ketamine in decreasing intracranial pressure in children with intracranial hypertension: Clinical article. J Neurosurg Pediatr 2009;4:40-6. https://doi.org/10.3171/2009.1.PEDS08319.

[38] Wang X, Ding X, Tong Y, Zong J, Zhao X, Ren H, et al. Ketamine does not increase intracranial pressure compared with opioids: meta-analysis of randomized controlled trials. J Anesth 2014;28:821-7. https://doi.org/10.1007/s00540-014-1845-3.

[39] Base de données publique des médicaments. KETAMINE PANPHARMA $250 \mathrm{mg} / \mathrm{mL}$, solution injectable (I.V.-I.M.) - Résumé des caractéristiques du produit n.d.

[40] Luginbühl M, Gerber A, Schnider TW, Petersen-Felix S, Arendt-Nielsen L, Curatolo M. Modulation of Remifentanil-Induced Analgesia, Hyperalgesia, and Tolerance by Small-Dose Ketamine in Humans: Anesth Analg 2003:726-32. https://doi.org/10.1213/01.ANE.0000048086.58161.18.

[41] Nesher N. Morphine With Adjuvant Ketamine vs Higher Dose of Morphine Alone for Immediate Postthoracotomy Analgesia 2009:245-52. https://doi.org/10.1378/chest.08-0246.

[42] Brinck E, Tiippana E, Heesen M, Bell RF, Straube S, Moore RA, et al. Perioperative intravenous ketamine for acute postoperative pain in adults. Cochrane Database Syst Rev 2018. https://doi.org/10.1002/14651858.CD012033.pub4.

[43] Motov S, Rockoff B, Cohen V, Pushkar I, Likourezos A, McKay C, et al. Intravenous Subdissociative-Dose Ketamine Versus Morphine for Analgesia in the Emergency Department: A Randomized Controlled Trial. Ann Emerg Med 2015;66:222-229.e1. https://doi.org/10.1016/j.annemergmed.2015.03.004. 
[44] Bhutta AT, Schmitz ML, Swearingen C, James LP, Wardbegnoche WL, Lindquist DM, et al. Ketamine as a neuroprotective and anti-inflammatory agent in children undergoing surgery on cardiopulmonary bypass: a pilot randomized, double-blind, placebo-controlled trial. Pediatr Crit Care Med J Soc Crit Care Med World Fed Pediatr Intensive Crit Care Soc 2012;13:328-37. https://doi.org/10.1097/PCC.0b013e31822f18f9.

[45] Loix S, De Kock M, Henin P. The anti-inflammatory effects of ketamine: state of the art. Acta Anaesthesiol Belg 2011;62:47-58.

[46] AFSSAPS. Douleur rebelle en situation palliative avancée chez l'adulte 2010.

[47] Chong C, Schug SA, Page-Sharp M, Jenkins B, Ilett KF. Development of a Sublingual/Oral Formulation of Ketamine for Use in Neuropathic Pain: Preliminary Findings from a Three-Way Randomized, Crossover Study. Clin Drug Investig 2009;29:317-24. https://doi.org/10.2165/00044011-200929050-00004.

[48] Niesters M, Martini C, Dahan A. Ketamine for chronic pain: risks and benefits. Br J Clin Pharmacol 2014;77:357-67. https://doi.org/10.1111/bcp.12094.

[49] Société Française d'Anesthésie et de Réanimation. Mise au point sur l'utilisation de la kétamine 2018.

[50] Cohen SP, Bhatia A, Buvanendran A, Schwenk ES, Wasan AD, Hurley RW, et al. Consensus Guidelines on the Use of Intravenous Ketamine Infusions for Chronic Pain From the American Society of Regional Anesthesia and Pain Medicine, the American Academy of Pain Medicine, and the American Society of Anesthesiologists: Reg Anesth Pain Med 2018:1. https://doi.org/10.1097/AAP.0000000000000808.

[51] Andrade C. Ketamine for Depression, 3: Does Chirality Matter?: (Clinical and Practical Psychopharmacology). J Clin Psychiatry 2017;78:e674-7. https://doi.org/10.4088/JCP.17f11681.

[52] Kavalali ET, Monteggia LM. Synaptic mechanisms underlying rapid antidepressant action of ketamine. Am J Psychiatry 2012;169:1150-6. https://doi.org/10.1176/appi.ajp.2012.12040531.

[53] Prescrire Rédaction. Médicaments à écarter pour mieux soigner : bilan 2021- Rev Prescrire 2020;40(446):929-941 https://prescrire.org/Fr/202/1834/55640/0/PositionDetails.aspx.

[54] Inscription sur la liste des stupéfiants des préparations injectables à base de kétamine à compter du 24 avril 2017 - Point d'Information - ANSM : Agence nationale de sécurité du médicament et des produits de santé n.d. https://ansm.sante.fr/S-informer/Points-d-information-Points-dinformation/Inscription-sur-la-liste-des-stupefiants-des-preparations-injectables-a-base-deketamine-a-compter-du-24-avril-2017-Point-d-Information (accessed December 19, 2020).

[55] Observatoire français des drogues et toxicomanies. Substances psychoactives, usagers et marchés : les tendances récentes (2017-2018) 2018.

https://www.ofdt.fr/publications/collections/periodiques/lettre-tendances/substancespsychoactives-usagers-et-marches-les-tendances-recentes-2017-2018-tendances-129-decembre2018/

[56] Observatoire français des drogues et toxicomanies. Tendances Récentes et Nouvelles Drogues. Rapport Marseille/PACA 2018 2018. https://www.paca.ars.sante.fr/system/files/201912/rapport\%20TREND\%20Marseille-PACA\%202018.pdf

[57] Cheng W-C, Dao K-L. The Emergence of Deschloro-N-ethyl-ketamine, a Ketamine Analog, in Drug Seizures and Drug Driving Cases in Hong Kong. J Anal Toxicol 2020;44:886-95. https://doi.org/10.1093/jat/bkaa038.

[58] Larabi IA, Zerizer F, Ameline A, Etting I, Joseph D, Kintz P, et al. Metabolic profiling of deschloro- $N$ - ethyl- ketamine and identification of new target metabolites in urine and hair using human liver microsomes and high- resolution accurate mass spectrometry. Drug Test Anal 2021:dta.3007. https://doi.org/10.1002/dta.3007.

[59] Morris H, Wallach J. From PCP to MXE: a comprehensive review of the non-medical use of dissociative drugs. Drug Test Anal 2014;6:614-32. https://doi.org/10.1002/dta.1620.

[60] Freese TE, Miotto K, Reback CJ. The effects and consequences of selected club drugs. J Subst Abuse Treat 2002;23:151-6. https://doi.org/10.1016/s0740-5472(02)00267-2.

[61] Drugs involved in drug-facilitated crimes: Part I: Alcohol, sedative-hypnotic drugs, gammahydroxybutyrate and ketamine. A review | Elsevier Enhanced Reader n.d. https://doi.org/10.1016/j.pharma.2010.05.002. 
[62] Larabi IA, Fabresse N, Etting I, Nadour L, Pfau G, Raphalen JH, et al. Prevalence of New Psychoactive Substances (NPS) and conventional drugs of abuse (DOA) in high risk populations from Paris (France) and its suburbs. Drug Alcohol Depend 2019;204:107508. https://doi.org/10.1016/j.drugalcdep.2019.06.011. 

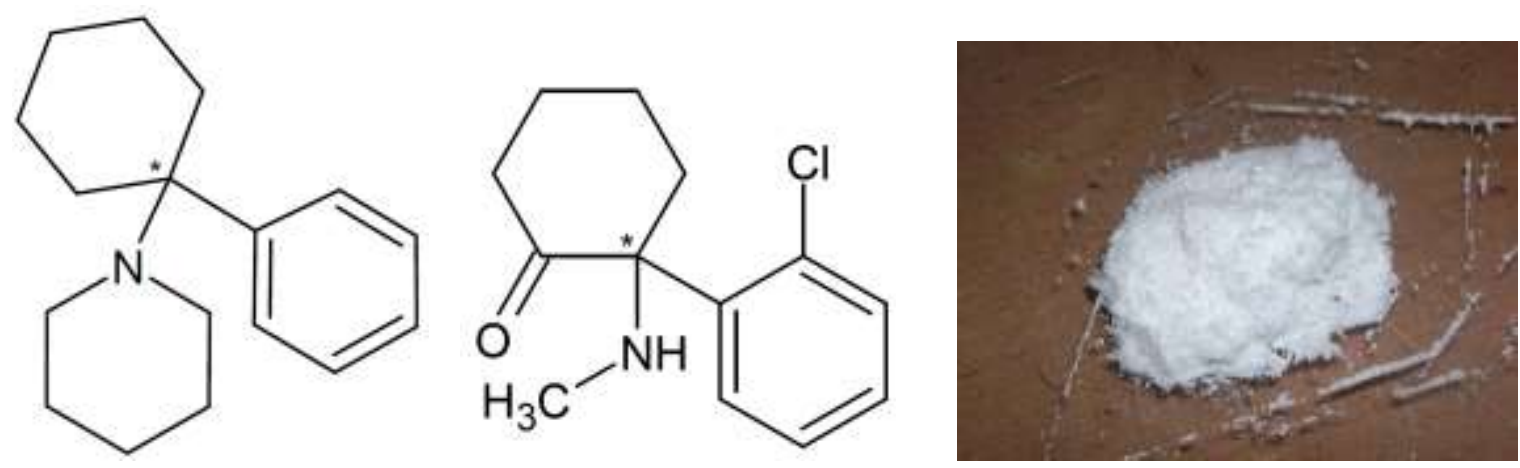

Figure 1 : A gauche : structure chimique de la molécule de phencyclidine. Au centre : structure chimique de la molécule de kétamine. A droite : cristaux de kétamine (* : carbone asymétrique).

Figure 1: Left: chemical structure of the phencyclidine molecule. Center: chemical structure of the ketamine molecule. Right: ketamine crystals (*: asymmetric carbon). 
Tableau 1 Principales indications de la kétamine et de l'eskétamine en France. Main indications for ketamine and esketamine in France.

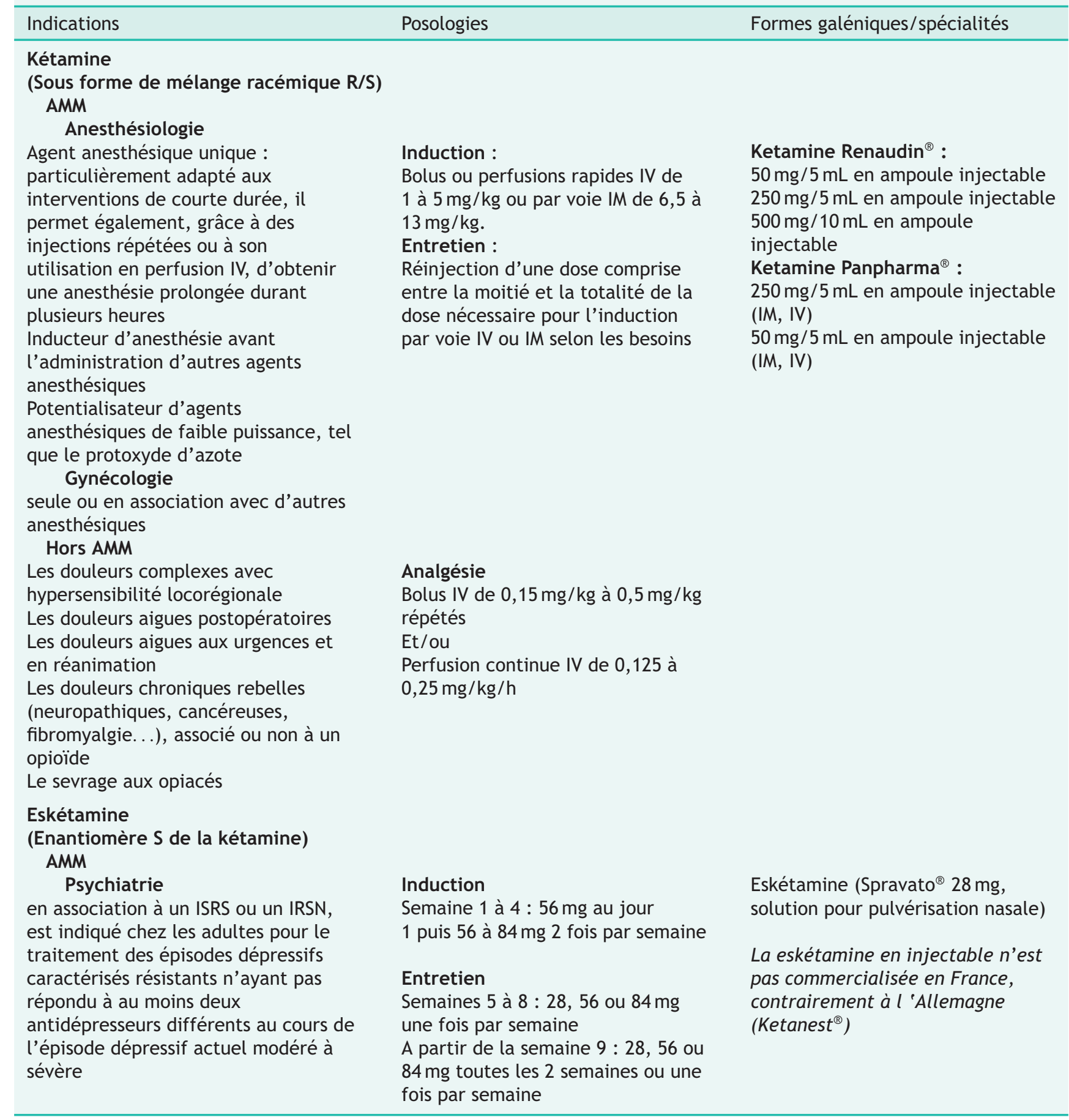

